\title{
Exclusão digital: como é estar do lado errado da divisão digital ${ }^{1}$
}

\section{Digital exclusion: be on the wrong side of the digital divide}

Massimo Ragnedda² e Maria Laura Ruiu³

Tradução do inglês: Andrea Limberto. andrealimberto@gmail.com.

2 Professor da Universidade de Northumbria (Inglaterra), com doutorado em Comunicação e Estudos Interculturais pela Universidade de Sassari (Itália). Foi pesquisador visitante de diversas instituições, entre elas: Institute of Communication Studies (University of Leeds, 2003-2004), Cambridge University (2006-2007) e Oxford Internet Institute (Oxford University). massimo.ragnedda@northumbria.ac.uk. 


\section{Resumo}

O desenvolvimento da sociedade da informação reforçou a existência de obstáculos que dificultam o acesso e o uso apropriado das tecnologias por certos grupos, levando a novas formas de exclusão no mercado de trabalho, nas instituições governamentais, no lazer, e nas atividades educativas. Contudo, reduzir o hiato entre aqueles que estão conectados e aqueles que não estão tendo acesso físico mais barato e mais rápido à internet não resulta automaticamente numa eliminação da distância colocada pelas desigualdades digitais. É um erro assumir um posicionamento tecnologicamente determinista, que vê o acesso à tecnologia como solução para os problemas sociais, incluindo problemas de desigualdade social, democracia, liberdade, interação social e um senso de comunidade. Na verdade, muitas dimensões e padrões existentes podem gerar e reforçar desigualdades, aumentando ainda mais as distâncias entre cidadãos/usuários. O termo "hiato digital", muitas vezes usado como expressão binária, pode não ser adequado porque sugere um hiato unidimensional, baseado principalmente no fator econômico - possuir tecnologia -, ao passo em que há hiatos em perspectivas múltiplas, que vão além do simples acesso ou da obtenção dos recursos. Essas dimensões criam desigualdades digitais que, se não forem retratadas, produzem e reforçam as desigualdades sociais. Os conceitos de estratificação social e digital estão intimamente interligados.

Palavras-chave

Hiato digital, inclusão digital, exclusão digital, desigualdade digital. 
Abstract

The development of the information society has highlighted the existence of obstacles preventing certain social groups from accessing and properly using technologies, leading to new forms of exclusion from the job market, governmental institutions, leisure and academic activities. However, reducing the gap between those who connect and those who do not by offering cheaper and faster physical access does not automatically translate into closing the gap in terms of digital inequalities. The technological determinist position, which sees access to technology as being able to solve social problems, including problems of social inequality, democracy, freedom, social relationships and sense of community, is misleading. In fact, several dimensions and patterns can generate and reinforce inequalities, further increasing the distances between citizens/users. The term "digital divide", often used as a binary expression, is confusing, because it suggests a onedimensional gap, mainly based on the economic factor - possession of technologies -, while there are gaps in multiple dimensions that go beyond the simple access to or possession of resources. These dimensions create digital inequalities that, if not mirrored, can produce and reinforce social inequalities. The concepts of social and digital stratification are intimately intertwined.

\section{Keywords}

Digital divide, digital inclusion, digital exclusion, digital inequalities. 
A sociedade contemporânea pode ser representada como uma rede digital na qual algumas das atividades humanas e sociais mais importantes acontecem. A exclusão do acesso ou o acesso limitado ao ambiente digital podem ser uma fonte significativa de desigualdade social. A partir disso, a posição assumida na sociedade em rede, em que informações econômica e socialmente relevantes circulam, pode ser interpretada como um fator produtor de desigualdades. Essa ideia está alinhada com uma visão do conceito de desigualdade que se relaciona ao ambiente digital e à inclusão/exclusão social. Por exemplo, ela é evidente, embora implicitamente, na política da Comissão Europeia em relação à inclusão digital, que a denomina como "e-inclusão" (COMISSÃO EUROPEIA, 2005). Desde o início dos anos 2000 essa expressão tem sido um dos três pilares estratégicos do plano da Comissão Europeia i2010 (MILLARD, 2006). A estratégia europeia é garantir que pessoas - incluindo aquelas que estão em desvantagem em termos de habilidades, localização geográfica, disponibilidade de recursos, educação, idade, gênero, e grupo étnico - possam acessar e se beneficiar da sociedade da informação (COMISSÃO EUROPEIA, 2005).

Uma das pressuposições deste artigo é que as desigualdades digitais, se não forem reduzidas, podem aumentar ainda mais as diferenças socioeconômicas. Governos, em particular, realizam um papel fundamental na redução das desigualdades experimentadas on-line. Essas desigualdades no uso das tecnologias da informação e comunicação (TICs) não estão somente baseadas em fatores econômicos, mas envolvem múltiplas dimensões, como o acesso técnico, a autonomia, o suporte social, as habilidades, e formas de uso, que vão além do simples acesso ou da detenção dos meios. Essa perspectiva leva em conta os padrões relacionados aos estratos sociais. Deveríamos, então, sublinhar tanto os riscos como as oportunidades de uma sociedade digital caracterizada pela crescente importância do conhecimento e pelo incessante crescimento e pervasividade das TICs. Além disso, a convergência digital entre computadores, telecomunicações e indústrias de mídia fez que fosse necessário pensar maneiras de evitar a exclusão ou a discriminação digital. Embora as características de 
inclusão e abertura que marcaram a internet desde sua concepção tenham alimentado o mito de uma rede democrática e igualitárias, formas significativas de desigualdade não apenas persistem, mas são crescentes.

Como veremos, uma correlação entre exclusão social e digital existe. Ser excluído digitalmente significa estar de fora de áreas importantes da vida cotidiana. Como consequência disso, aqueles que estão aquém quanto ao capital digital - geralmente pessoas de classes e status sociais mais baixos - podem ficar presos na base de uma estrutura social. Este artigo tenta lançar luz sobre esse processo, destacando, em primeiro lugar, como a posição individual no estrato social e sua motivação influenciam a adoção e novas tecnologias. Em segundo lugar, veremos como o hiato digital, na base do processo de inclusão/ exclusão digital, não pode ser reduzido a uma simples questão de acesso à internet, mas é um fenômeno muito mais complexo. Em terceiro lugar, este artigo se concentra sobre a ideia do engajamento digital como um aspecto vital do processo de inclusão digital e a internet como uma ferramenta de inclusão e/ou exclusão. Por fim, antes de apresentar algumas conclusões, este artigo irá sublinhar consequências negativas para os indivíduos que se localizam do lado desfavorecido do hiato digital.

\section{Falta de estímulo}

A interação entre fatores de diferenciação social e cultural, por um lado, e formas de socialização digital, por outro, fazem emergir formas específicas de desigualdade que marcam fortemente as possibilidades na vida de um indivíduo. As diferenças no uso da tecnologia digital não podem ser explicadas simplesmente com base no "capital cultural" ou no "capital de conhecimento acumulado" dos indivíduos. Somado a esses elementos, nós devemos considerar a posição individual no estrato social e sua motivação e direcionamento no uso da internet. A inclusão digital e os tipos de atividades realizadas na internet são, dessa forma, moldados não somente a partir das habilidades que os usuários possuem, mas também pelo interesse - ou falta dele - em usar as tecnologias digitais e pela 
posição individual na estrutura social. A falta de interesse cria uma forma de autoexclusão pela qual a possibilidade de escolha individual aparece como uma ilusão. Motivação e estímulo são muitas vezes dados a partir das normas e valores culturais em que o indivíduo está inserido, estruturando as experiências da vida cotidiana. O conceito de habitus (BOURDIEU, 1984) pode ser útil nesse ponto. O termo se refere ao processo de reprodução cultural e sua habilidade de gerar comportamentos regulares que afetam a vida social, as habilidades e as disposições que os indivíduos adquirem a partir de suas experiências de vida. De acordo com Bourdieu, habitus reúne crenças, interesses, pensamentos, gostos e um entendimento de cada indivíduo em relação ao mundo que o circunda. Tratase da encarnação do capital cultural que influencia tanto as ações individuais quanto a construção de seu mundo social, somado à influência do mundo externo para resultar na possibilidade de escolha individual. A interação entre os mundos interno e externo cria um habitus único para cada pessoa, e esse habitus específico influencia a escolha individual na adoção ou não das tecnologias.

Em outras palavras, a falta de interesse ou falta de estímulo na adoção das TICs não são "neutras", ou um simples caso de preferência individual, mas reproduzem outros fatores socioeconômicos e culturais presentes no sistema social. Essas desigualdades são também reproduzidas on-line e afetam as oportunidades dos cidadãos, podendo aumentar ou diminuir as possibilidades que eles têm de ascender em hierarquias sociais. $\mathrm{O}$ acesso tanto quanto o uso das tecnologias de informação (TI) estão na base da participação e nas vantagens de uma sociedade baseada na informação. Estar excluído ou ter acesso e uso limitado às TICs significa não ter os requisitos necessários no kit para melhorar de condição de vida. Isso é parte de um quadro maior que inclui os efeitos das desigualdades digitais produzidas pela digitalização - ou a reprodução digital das desigualdades (HARGITTAI, 2004; WESSELS, 2013). O capital digital e a entrada digital de cada indivíduo têm papel crucial numa ampla gama de desdobramentos da vida social. Como Robinson et al. (2015) corretamente pontuam, aqueles que usam a internet de modo eficiente e fazem parte de uma vida social mediada têm 
vantagens sobre aqueles que estão em desigualdade digital. Já que vida digital e vida social estão cada vez mais conectadas entre si - e suas diferenças tendem a se apagar -, uma participação plena no ambiente digital acaba por afetar nada menos que aspectos cruciais da qualidade de vida individual, como obtenção de recursos e acesso ao mercado de trabalho, lazer, oportunidades acadêmicas, e aquisição de informações relevantes. Estar em vantagem social significa também usar a internet com mais frequência e de maneira diferenciada, desenvolvendo diferentes níveis de habilidade que, por sua vez, produzem resultados concretos tanto no âmbito digital quanto social.

A disponibilidade de acesso físico, cognitivo e motivacional em relação à internet afeta não somente o nível de satisfação e as possibilidades do usuário, mas representa também uma condição favorável para o processo de aquisição de capital digital. Os eixos tradicionais de desigualdade social tendem a se reproduzir on-line, refletindo-se também no nível de habilidade na rede (DEURSEN; HELSPER; EYNON, 2014). Todos esses aspectos estão relacionados com as capacidades individuais em melhorar sua qualidade de vida a partir da mobilidade social.

Está cada vez mais claro que as relações entre os indivíduos e o capital digital representam um aspecto central em termos de alcançar sucesso pessoal e profissional. Aqueles que são mais estimulados, possuem mais capital digital e estão mais envolvidos na vida digital tendem a conseguir maiores benefícios do que aqueles que não participam ativamente da vida social digital. Isso significa que diferentes experiências on-line geram diferentes capacidades e oportunidades de produzir bens econômicos e culturais a serem investidos num mercado voltado para o digital, potencialmente para melhorar as condições de vida de uma pessoa. Em resumo, uma das principais pressuposições deste artigo é que as experiências digitais influenciam os desdobramentos na vida e no sistema de desigualdade. Para entender melhor o que estamos interpretando por experiência digital, há a necessidade de aprofundar o conceito de "hiato digital". O próximo subitem deste trabalho mostrará como o hiato digital indica 
não uma separação nos termos de uma divisão binária, mas um continuum em diferentes níveis de apropriação e uso das tecnologias de informação.

\section{Hiato digital}

A literatura científica enfatiza que a internet oferece muitas vantagens em termos de engajamento político, participação cívica e na moldagem da opinião pública. Contudo, parece-nos que a estratificação off-line também influencia fortemente as atividades on-line. Importantes inovações relacionadas à paisagem social estão alterando os significados e as manifestações das desigualdades sociais. A maneira como as novas tecnologias são utilizadas e incluídas na sociedade e a posição individual no sistema social afetam a determinação dos recursos que podem ser acessados. Ao mesmo tempo, os recursos individuais determinam quando e como os usos das tecnologias são apropriados. A divisão digital se refere não somente à disponibilidade de computadores e à possibilidade de acesso à World Wide Web, mas também às habilidades e às motivações necessárias para seu uso. O fato de que a disponibilidade da internet tem crescido progressivamente não significa necessariamente que as desigualdades digitais/sociais foram reduzidas, mas é mais provável que ocorra o oposto. Na verdade, enquanto o espraiamento da internet segue os modelos tradicionais de difusão tecnológica, chegando cada vez a mais cidadãos/usuários, isso não aumenta a capacidade de as pessoas usarem as tecnologias de maneira eficiente e ampliarem seu capital digital, social e cultural.

É necessário repensar o hiato digital para além da simples dicotomia entre o que a internet tem e não tem (HARGITTAI, 2000; 2002; NORRIS, 2001; DIMAGGIO et al., 2001; CASTELLS, 2004; ROGERS, 2003; BARZILAI-NAHON, 2006; RAGNEDDA; MUSCHERT, 2016) e refletir sobre outras variáveis que possam exacerbar as desigualdades digitais e a exclusão digital. Nesse sentido, parece mais apropriado analisar o fenômeno em relação à qualidade do uso (BENKLER, 2006), às capacidades e às habilidades digitais dos cidadãos com diferentes bagagens socioeconômicas (HARGITTAI, 2002; 2010; DEURSEN; DIJK, 2009), e às diferentes maneiras pelas quais as TICs são utilizadas (HARGITTAI; HSIEH, 
2010). As desigualdades no uso da internet são influenciadas por diversos fatores, que Warschauer (2003) agrupa em quatro categorias: recursos humanos, físicos, digitais, e sociais - recursos que moldam a maneira com que os usuários se relacionam com a tecnologia. Essa é a razão pela qual o estudo das desigualdades digitais começou a se voltar para a análise desse tipo de fatores, mais do que simplesmente considerar seu aspecto tecnológico. Enquanto a falta de acesso à internet tem progressivamente diminuído no curso das últimas décadas, ao menos na sociedade ocidental, os benefícios advindos daí não são comumente experimentados por todos os usuários (ATTEWELL, 2001; HOWARD; BUSCH; SHEETS, 2010; ONO; ZAVODNY, 2003), e a utilização da tecnologia resulta em um crescente abismo (HILBERT; LÓPEZ; VÁSQUEZ, 2010).

Seria um engano pensar que o acesso dos indivíduos à internet, bem como seu uso, pode automaticamente converter e transformar habilidade e capital digital em outros desdobramentos sociais. Ao mesmo tempo, podemos argumentar que, numa sociedade facilitada digitalmente, a capacidade/ habilidade, a motivação, a educação, e a "qualidade" da informação adquirida on-line influencia as oportunidades obtidas no âmbito social. Estar excluído ou apenas parcialmente envolvido no âmbito on-line pode influenciar o capital digital e, em decorrência, influenciar efeitos na vida. Já que a internet está evoluindo e se tornando cada vez mais vital nas nossas atividades cotidianas, formas de exclusão - tanto social como digital - também tendem a emergir. Ao analisar essas formas de exclusão ou desvantagem, os contextos e históricos social, econômico e político não podem ser ignorados: eles interferem no acesso e no uso da internet, e essa experiência on-line exerce influência nas oportunidades e possibilidades de vida que os indivíduos têm no mundo off-line.

O hiato e as desigualdades digitais tendem não somente a reforçar o desequilíbrio digital já existente na sociedade off-line, mas também a aumentar a distância entre os mais e os menos favorecidos. Os estratos sociais que tendem a obter mais recursos de valor são os mesmos que conseguem fazer uso das TICs de maneira mais vantajosa. Por um lado, a internet oferece um sem número de 
oportunidades; por outro, ela não pode ser vista como geradora de igualdades. Na verdade, usos diferentes podem contribuir para determinar desigualdades tanto no âmbito social quanto digital. Isso significa que as variáveis socioeconômicas e culturais na base das desigualdades sociais interferem na qualidade das experiências on-line, afetando assim a quantidade e o tipo dos possíveis benefícios. Na verdade, enquanto essas oportunidades e recursos valorosos estão teoricamente ao alcance de todos, eles são invisíveis para algumas pessoas. Em outras palavras, as possibilidades que a internet oferece aos cidadãos em termos econômicos, políticos, sociais, e culturais não são usufruídas por todos da mesma forma. As classes sociais que gozam de privilégios sociais tendem a se tornar ainda mais privilegiadas. A internet interfere nas possibilidades que um cidadão tem para melhorar sua condição de vida, mas o processo repete um ciclo vicioso, baseado numa posição social/econômica/cultural inicial. É isso o que Ragnedda (2017) define como o terceiro nível do hiato digital: a separação na interação entre posições off-line socialmente privilegiadas e as desigualdades digitais em relação aos recursos socialmente relevantes que esperamos ganhar com a internet. Contudo, já que as desigualdades sociais e digitais são tão fortemente interligadas, uma análise dos contrastes digitais deve levar em conta o sistema cultural e social no qual as tecnologias são incluídas.

A divisão digital é apenas uma das dimensões de um sistema mais amplo de desigualdades. A desigualdade no acesso à internet continua a existir e está de acordo com a estratificação social, determinando áreas de exclusão social. O acesso universal, que elimina a primeira forma de hiato digital - em termos de quem possui internet ou não- está longe de ser uma garantia. E mesmo que chegássemos a um ponto de saturação de provimento de acesso, ele não seria suficiente para suprimir as desigualdades digitais. Formas diferentes de desigualdade seriam perpetuadas, e diferentes níveis de desigualdade, reforçados. Indivíduos de classes sociais distintas e de diferentes status podem ter o mesmo acesso à internet, em termos de conexão física, mas apenas alguns privilegiados conseguem converter esse acesso físico em benefício social. 


\section{Podemos emendar o hiato digital?}

A literatura científica destaca duas principais abordagens teóricas em relação às desigualdades digitais, que são representadas pela "normalização" e pela "estratificação". A primeira abordagem, conhecida também como standardization, se concentra na ideia de que a disparidade atual será gradualmente resolvida graças à crescente oferta da tecnologia a menores custos e com interfaces mais simples, o que levaria a um nivelamento geral. Embora o abismo no acesso à internet tenha começado a ser percebido como uma causa potencial de exclusão social (CHINN; FAIRLIE, 2006; FOX, 2005), muitos acadêmicos e, mais importante, muitos políticos ainda não atentaram para as consequências sociais relacionadas ao crescimento do hiato digital (COMPAINE, 1988; 2001; ESTADOS UNIDOS, 2004; THIERER, 2000) - ou as subestimam. Eles defendem que, como o que ocorreu com outras tecnologias no passado, a distância entre aqueles que têm acesso à internet e aqueles que não têm vai eventualmente desaparecer, num certo ciclo histórico. Os abismos tecnológicos desapareceriam gradualmente graças ao declínio no custo da tecnologia, resultando no apagamento dos problemas que envolvem o hiato digital (COMPAINE, 1988; 2001); a solução seria apenas uma questão de tempo, porque a tecnologia irá se adaptar ao mercado (THIERER, 2000).

Assim, muitas das diferenças que podem ser detectadas no uso das TICs e no acesso a elas não deveriam necessariamente ser base para uma preocupação social. Alguns autores argumentam que o problema da desigualdade no uso das TICs é superestimado. Compaine (2001) defende que certos segmentos da população não usam a internet como outros. Esse fato é parte de uma diferença no tempo de adoção da tecnologia que caracteriza o estágio inicial da introdução de qualquer inovação. Esses atrasos seriam tipicamente cíclicos, normalmente superados quando o desenvolvimento tecnológico chega a um ponto em que os mecanismos do mercado não têm mais que arcar com os custos, e o conhecimento técnico dos usuários mais antigos pode ser repassado (COMPAINE, 2001). A taxa de difusão do uso da internet é muito maior do que, 
por exemplo, a do rádio e da televisão. Contudo, da mesma forma que se viu com o uso da televisão, de geladeiras, de máquinas de lavar, e de carros, é esperado que uma desigualdade inicial de acesso seja remediada com o tempo. Em essência, o atual desnível seria apenas temporário e tenderia a desparecer com o tempo. Enquadrar o hiato digital como um problema tecnológico, como temos visto, sugere que o acesso à tecnologia é capaz de corrigir problemas sociais existentes, como desigualdades e questões relacionadas à democracia, à liberdade, às relações sociais, e à vida comunitária.

A segunda hipótese, aquela da estratificação, defende que as desigualdades nascidas com a introdução das TICs serão somadas àquelas já existentes, num processo cumulativo e cíclico. Os grupos que foram mais vagarosos na adoção das novas tecnologias nunca conseguirão vencer a distância em relação àqueles mais rápidos, com o consequente crescimento do acesso e usos diferenciados. Muito provavelmente a questão da distância em relação ao acesso irá diminuir; no entanto, a existência de um nível diferente de desigualdades, derivado das diferenças na forma de uso das TICs, sugere que a condição de "normalização" não é alcançada, mas o que se tem é uma reconfiguração da estratificação social. Essa hipótese se refere à possibilidade de que o hiato digital será parte de uma estrutura social já muito polarizada nas esferas econômica, cultural, e social - que assim as desigualdades existentes serão exacerbadas. Como resultado, aqueles que se encontram numa posição de relativa vantagem acabam por consolidar e possivelmente aumentar seus privilégios, em detrimento da categoria de consumidores que foram mais vagarosos em adotar as novas tecnologias - e assim não será possível eliminar essa diferença jamais.

No entanto, ao longo dos últimos anos, uma atitude equidistante entre as duas hipóteses apresentadas tem ganhado proeminência. De acordo com essa posição intermediária, ambos os caminhos são parcialmente válidos e nenhum deles dá conta de explicar o fenômeno completamente. Diante da multiplicidade das diferenças apontadas, é possível imaginar que a distância no acesso à tecnologia pode ser superada em algum momento, mas enquanto isso, outras desigualdades 
em termos de habilidades, de motivação, de capital, e de capacidade de aproveitar as oportunidades da internet são perpetuadas. É importante lembrar que embora as diferenças na adoção das TICs entre diferentes classes sociais sejam parcialmente amenizadas, elas se mantêm em relação a alguns estratos sociais, como entre os indivíduos mais velhos, cidadãos com rendas menores, alguns grupos étnicos e entre aqueles com um baixo nível educacional e que batalham para acessar - no sentido mais amplo do termo, ou seja, não meramente no aspecto físico -, para usar e aproveitar as vantagens da internet. Há uma tendência nos estratos sociais mais privilegiados de acumular as oportunidades advindas de diferentes níveis de acesso e de usos das TICs. Mesmo que o hiato pareça advir dos diferentes níveis de acesso, fica claro que para atingir todo o potencial das TICs é necessário chegar a certos níveis de capital digital, e isso depende, por um lado, das características da tecnologia que está sendo usada e, por outro, do capital social, cultural, e político que os cidadãos/usuários possuem.

As desigualdades digitais tendem a minimizar as desigualdades sociais, culturais, econômicas e políticas preexistentes nas sociedades. Demanda-se continuamente que usuários e cidadãos melhorem, atualizem e potencializem suas habilidades, de maneira a poderem "manejar" um volume excessivo de informações (DIJK; HACKER, 2003, p. 316) - e, mais importante, a se tornarem cidadãos completos. Já que a infraestrutura vital para o desenvolvimento apropriado da sociedade é movimentada on-line, torna-se crucial que os cidadãos estejam aptos a acessar fisicamente a internet, mas que possam habilmente navegar no mundo cibernético. Isso não é só uma questão de alfabetização ou de habilidade digital, isto é, um conjunto de competências necessárias para operar computadores e redes, pesquisar e selecionar informações digitais e usálas para atingir objetivos específicos (DIJK, 2005) - mas também é necessário ter a possibilidade de se sentir parte da e-comunidade, de serem e-cidadãos e estarem incluídos numa sociedade digital.

Para esclarecer melhor, não pretendemos negar a importância da revolução trazida com o advento da internet. Acreditamos que ela possibilitou 
novas formas de relação social, revolucionou o acesso à informação e até mesmo permitiu que certas áreas do mundo pudessem lutar por democracia, ainda que o efeito nesse sentido tenha sido muitas vezes superestimado, como no caso da Primavera Árabe. No entanto, o rápido crescimento da internet não acontece de maneira uniforme, seja no nível global ou nos Estados individualmente. Áreas menos desenvolvidas do mundo não têm conseguido participar desse crescimento, em virtude da pobreza da população, da falta de infraestrutura, das restrições políticas, e da falta de uma cultura tecnológica e da motivação em adotar novas tecnologias de comunicação. Dessa forma, a disseminação das TICs está redesenhando os mapas da pobreza e da riqueza, aumentando as diferenças entre o Norte e Sul globais, e gerando novas áreas de exclusão mesmo nas nações mais desenvolvidas. Inclusive dentro dos próprios Estados existem diferenças na adoção e no uso da internet, assim como nas capacidades de reinvestir ganhos obtidos on-line - em termos de informações e uso de recursos relevantes - no sentido de melhorar socialmente. Essas desigualdades determinam diferenças importantes tanto entre nações - hiato digital global quanto entre as pessoas dentro dos próprios Estados - hiato digital democrático.

\section{Engajamento digital}

Os aspectos individuais e sociais dos sujeitos determinam quais recursos estão disponíveis para eles. Em resposta, os recursos disponíveis afetam o acesso e atuam como uma base sobre a qual novas desigualdades digitais se desenvolvem. Sua distribuição desproporcional produz desigualdades também no aceso às tecnologias digitais, e, em decorrência, produz a primeira forma de exclusão - o primeiro nível do hiato digital. A desigualdade de acesso à internet também depende de características da tecnologia e de diferentes formas de apropriação tecnológica, que resultam em diferenças de habilidade e, assim, em novas formas de exclusão - o segundo nível do hiato digital. A somatória das desigualdades consideradas aqui inibe a possibilidade de participação plena e de inclusão digital. A apropriação da tecnologia tende a influenciar o nível 
de participação social. Os índices que ilustram a posição de um indivíduo na sociedade podem ser "variáveis individuais" - idade, gênero, grupo étnico ou "variáveis sociais" - renda, inserção no mercado de trabalho, status social. Essas variáveis influenciam a maneira pela qual nós acessamos e usamos os recursos que estão na base do processo de exclusão da sociedade. A exclusão digital e a exclusão social estão inevitavelmente relacionadas e se influenciam reciprocamente. O acesso e o uso da internet de uma maneira efetiva podem ampliar as oportunidades, melhorar a qualidade de vida e reforçar a posição privilegiada de alguns grupos na sociedade - o terceiro nível do hiato digital. Por comparação, aqueles que não usam a internet "de maneira efetiva" estão propensos a perder oportunidades de vida nas esferas econômica, política, educacional, cultural, relacional e social (DIJK, 2005). Em outras palavras, acesso e uso limitado à internet afetam as oportunidades dos cidadãos (DIMAGGIO et al., 2004) e explicam como e por que o acesso individual à internet é importante para o processo de inclusão social (WARSCHAUER, 2003).

É importante também sublinhar que os estratos sociais da população estão mais expostos às incertezas do empobrecimento digital. Isso inclui as pessoas mais velhas, os indivíduos que não possuem um emprego ativo, como desempregados, trabalhadores inativos, pensionistas; indivíduos caracterizados por baixo nível educacional e com limitado capital cultural; indivíduos que vivem em áreas geográficas menos desenvolvidas, como áreas rurais, ou em países menos desenvolvidos - hiato global. Esses grupos em desvantagem, que já sofrem com desigualdades no sistema social, também estão sofrendo com as desigualdades na arena digital. Eles estão alguns passos atrás enquanto o resto do mundo se movimenta para frente (DIMAGGIO et al., 2004, p. 368). Nesse ponto, o paralelo entre as desigualdades sociais e as digitais emerge muito claramente. Ademais, o enriquecimento dos sujeitos de maneira dinâmica e cumulativa privilegia aqueles que já possuem mais habilidade e que têm mais experiência, enquanto os mecanismos de empobrecimento para aqueles que estão menos familiarizados com as TICs estão aumentando os índices de desigualdade 
na sociedade. É algo parecido com o que o "efeito Matthew" prevê em relação ao advento da disseminação da tecnologia: aqueles que têm mais experiência na administração das novas tecnologias e fazem uso mais variado delas vão se beneficiar mais (RAGNEDDA; MUSCHERT, 2016). Isso evidentemente tem efeitos não somente em termos de habilidades tecnológicas, mas também em termos de um posicionamento social e econômico e na aquisição cultural.

\section{A internet: ferramenta de inclusão ou instrumento de exclusão?}

As pessoas que ocupam um status mais baixo socialmente tendem a sofrer em termos de aquisição de habilidades digitais e, consequentemente, tendem a estar digitalmente e socialmente em desvantagem. A comunicação digital tem um papel importante no processo de estruturação da sociedade contemporânea e pode resultar na emergência de novas formas de exclusão social relacionadas às desigualdades digitais. A renda per capita não é o único dado considerado para a adoção das TICs, que também está relacionada à "qualidade" da informação encontrada on-line. Alguns usuários parecem estar menos interessados em procurar informações que estejam orientadas a um objetivo, por exemplo, como aqueles pertencentes a grupos menos favorecidos econômica e socialmente (SELWYN; GORARD; FURLONG, 2005), que se concentram na busca por informações mas sem propósito específico.

O hiato digital, dessa maneira, é uma forma de exclusão social e digital que depende não somente de fatores tecnológicos, demográficos e geográficos, mas também se relacionam a circunstâncias econômicas, culturais e sociais baseadas numa estrutura social existente. Todos esses fatores influenciam não somente o uso da internet, mas também sua estruturação e evolução cultural (RAGNEDDA; MUTSVAIRO, 2016). Como pudemos ver, o hiato digital se transforma ao longo do tempo de acordo com a difusão da inovação tecnológica. Faz-se necessário que ele seja constantemente redefinido, usando indicadores operacionais mais apropriados. A internet é um espaço que proporciona condições favoráveis para o crescimento do conhecimento como 
um bem comum. A estrutura da rede independe das fronteiras dos variados Estados e tem o potencial de conectar todos os habitantes da terra, reduzindo disparidades de todos os tipos. No entanto, independente dessas qualidades únicas, muitos obstáculos impedem uma integração completa. A assunção errônea de que existe uma forma de homogeneidade a partir do acesso não somente está incorreta, mas acaba por obscurecer e ocultar efeitos sociais, econômicos e culturais produzidos pelas diferenças no acesso e no uso das TICs. A exclusão digital - falta ou acesso limitado às tecnologias digitais - ou a discriminação digital - baseada na inabilidade para o uso das TICs - aumentam o risco de exclusão social.

Van Dijk (2005, p. 166-177) identifica a participação e a inclusão social como os fatores mais importantes no combate à desigualdade digital. Ele descreve algumas das áreas mais importantes em que a "exclusão digital" pode aparecer: economia, capacitação, sociedade, espacialidade, política, e institucional. Numa sociedade em rede em que os novos meios estão adquirindo papel cada vez mais importante nos benefícios e na competição entre os indivíduos, a mídia eletrônica convencional e outras formas tradicionais de comunicação não serão suficientes para possibilitar uma participação plena em cada uma dessas áreas, enquanto o uso da internet será cada vez mais vital.

\section{Estar do lado errado}

Na base da chamada "discriminação digital" está uma falta não só de conhecimento e de habilidade técnica no uso das TICs, mas também das habilidades sociais e da capacidade e motivação para criar uma rede que aprimore o capital social e a colaboração on-line. As desigualdades na vida digital podem atingir aspectos sociais, como a busca por emprego, compras, empreendimentos, acesso à saúde, à educação, socialização, engajamento político e padrões de consumo. Todos esses aspectos são determinados por um uso mais orientado da internet, baseado na capacidade de reinvestir informações relevantes de volta ao mercado, e afetando assim as oportunidades de vida dos indivíduos. 
A internet tem cada vez mais espaço na vida cotidiana, nas formas pelas quais a desigualdade social está em transformação. Desse modo, não fazer parte de uma vida digital pode ter efeitos significativos no percurso de vida de uma pessoa. Dentro de uma sociedade conectada, estar incluído nos fluxos de informação deveria ser algo central para as oportunidades estruturais que uma sociedade oferece para seus indivíduos, criando as condições para uma escolha consciente e livre para o uso das tecnologias digitais de forma a atingir um maior estado de acúmulo de riqueza. A participação na sociedade em rede, nesse sentido, deveria ser considerada como uma opção relacionada à percepção subjetiva de bem-estar, livre de restrições determinadas pela estrutura das desigualdades sociais. A igualdade está cada vez mais ligada à disponibilidade do conhecimento e da informação. A partir disso, a habilidade para manejar a tecnologia para obter esses conhecimentos se torna crucial. Nem todos podem ter a oportunidade de usar a informação - em termos de acesso físicos - ou está apto para acessá-la - em termos de habilidade. Ademais, nem todos os cidadãos conseguem utilizar a informação de uma maneira efetiva, de modo a satisfazer suas necessidades e desejos de melhorar suas oportunidades na vida. Estar do lado errado do hiato digital pode reforçar a chance de que algumas pessoas serão cidadãos de segunda ordem ou que não tenham cidadania de nenhuma maneira (DIJK, 2005).

As desigualdades no acesso e no uso da tecnologia não deveriam ser entendidas em termos de uma divisão preto no branco, mas com nuances de inclusão e de desigualdades no âmbito digital. A internet teoricamente aumenta as possibilidades na vida de todos os cidadãos. No entanto, ela tende também a reforçar alguns privilégios sociais, mais do que superá-los, promovendo os interesses daqueles que já possuem uma posição privilegiada na sociedade. Esses grupos são os que mais se beneficiam com a internet, porque têm melhor capital político, cultural, econômico, pessoal e social. É por esse motivo que quando analisamos o hiato digital e os processos de inclusão digital, é impossível não levar em consideração os contextos sociais e as necessidades dos potenciais usuários. 


\section{Conclusões}

Para concluir, queremos reiterar o que temos discutido ao longo deste artigo, notadamente que os benefícios no uso da internet operam como um ciclo vicioso baseado nas estruturas sociais, exacerbando desigualdades sociais. As capacidades e habilidades em encontrar, extrair e usar informações valiosas influenciam na condição social dos indivíduos. As diferenças na quantidade e na qualidade das oportunidades de educação das pessoas e o uso que fazem da mídia constituem uma dimensão social da desigualdade que tem recebido pouca atenção na tradição dos estudos de sociologia e de mídia. O advento dos novos meios abriu um vivo debate sobre as desigualdades no acesso às redes digitais, diferenças em relação a seu uso e no aproveitamento das oportunidades. Nesse cenário, articuladores de políticas públicas podem ter um papel crucial na tentativa de superar desigualdades não somente em termos de acesso, mas, mais importante, em termos de igualdade de oportunidades. A questão da desigualdade digital não é um problema tecnológico, e sim socioeconômico, e está enraizado na estratificação social. Assim, as políticas, em todos os níveis, das instituições locais a organizações internacionais, têm responsabilidade de promover e estimular a inclusão digital de forma a criar interfaces para o diálogo e alimentar a participação cidadã. Contudo, devemos lembrar que as políticas que levam as pessoas a utilizarem novas ferramentas podem ser consideradas como que se estivessem "forçando" uma mudança cultural e propagando uma "obrigação para a inclusão digital" ideológica. De modo a evitar a imposição de políticas de cima para baixo, educadores deveriam entender plenamente as reais necessidades dos cidadãos e equilibrá-las com aquelas do mercado de modo a oferecer as habilidades necessárias para o investimento num mercado regido pelo digital e melhorar suas condições de vida.

Por fim, cidadãos poderiam enxergar na expansão das novas tecnologias uma oportunidade concreta para melhorar de vida. Isso significa que atores políticos não deveriam somente propor uma capacitação básica tendo em vista aprimorar as habilidades digitais, mas também explicar o potencial das novas tecnologias em ajudar a melhorar a qualidade de vida de grupos em desvantagem. 


\section{Referências}

ATTEWELL, P. The first and second digital divides. Sociology of Education, Thousand Oaks, v. 74, n. 3, p. 252-259, 2001.

BARZILAI-NAHON, K. Gatekeepers, virtual communities and their gated: multidimensional tensions in cyberspace. International Journal of Communications, Law and Policy, London, v. 11, p. 1-28, 2006.

BENKLER, Y. The wealth of networks: how social production transforms markets and freedom. New Haven: Yale University, 2006.

BOURDIEU, P. Distinction: a social critique of the judgment of taste. Cambridge, MA: Harvard University, 1984.

CASTELLS, M. The power of identity. Malden: Blackwell, 2004.

CHINN, M. D.; FAIRLIE, R. W. The determinants of the global digital divide: a cross-country analysis of computer and Internet penetration. Oxford Economic Papers, Oxford, v. 59, n. 1, p. 16-44, 2007.

COMPAINE, B. M. Information gaps: myth or reality? In: . Issues in new information technology. Norwood: Ablex, 1988. p. 179-191.

. The digital divide: facing a crisis or creating a myth? Cambridge, MA: MIT, 2001.

DEURSEN, A. J. A. M.; DIJK, J. A. G. M. Improving digital skills for the use of online public information and services. Government Information Quarterly, Amsterdam, v. 26, p. 333-340, 2009. 
DEURSEN, A. J. A. M.; HELSPER, E. J.; EYNON, R. Measuring digital skills: from digital skills to tangible outcomes project report. 2014. Disponível em: <http:// bit.ly/2iXnmJb>. Acesso em: 19 ago. 2016.

DIJK, J. A. G. M. The deepening divide: inequality in the information society. London: Sage, 2005.

DIJK, J. A. G. M.; HACKER, K. The digital divide as a complex and dynamic phenomenon. The Information Society, London, v. 19, n. 4, p. 315-326, 2003.

DIMAGGIO, P. et al. Social implications of the Internet. Annual Review of Sociology, Palo Alto, v. 27, p. 307-336, 2001.

- Digital inequality, from unequal access to differentiated use. In: NECKERMAN, K. M. (Ed.). Social inequality. New York: Russell Sage Foundation, 2004. p. 355-400.

ESTADOS UNIDOS. Department of Commerce. Economics and Statistics Administration. National Telecommunications and Information Administration. A nation online: entering the broadband age. Washington, DC: Department of Commerce, 2004. Disponível em: <http://bit.ly/2jmr5yo>. Acesso em: 12 ago. 2016.

FOX, S. Digital divisions: there are clear differences among those with broadband connections, dial-up connections, and no connections at all to the Internet. Washington, DC: Pew Internet \& American Life Project, 2005. Disponível em: <http://pewrsr.ch/2kkTMJt>. Acesso em: 14 ago. 2016. 
HARGITTAI, E. Digital na(t)ives? Variation in Internet skills and uses among members of the "net generation". Sociological Inquiry, Hoboken, v. 80, n. 1, p. 92-113, 2010.

. Internet access and use in context. New Media \& Society, London, v. 6, n. 1, p. 137-143, 2004.

. Open portals or closed gates? Channeling content on the World Wide Web. Poetics, Amsterdam, v. 27, n. 4, p. 233-254, 2000.

- Second-level digital divide: differences in people's online skills. First Monday, [S.I.], v. 7, n. 4, 2002.

HARGITTAI, E.; HSIEH, Y.-L. P. From dabblers to omnivores: a typology of social network site usage. In: PAPACHARISSI, Z. (Ed.). A networked self: identity, community and culture on social network sites. London: Routledge, 2010. p. $146-168$.

HILBERT, M.; LÓPEZ, P.; VÁSQUEZ, C. Information societies or "ICT equipment societies"? Measuring the digital information-processing capacity of a society in bits and bytes. The Information Society, London, v. 26, n. 3, p. 157-178, 2010.

HOWARD, P.; BUSCH, L.; SHEETS, P. Comparing digital divides: Internet access and social inequality in Canada and the United States. Canadian Journal of Communication, Vancouver, v. 35, n. 1, p. 109-128, 2010.

COMISSÃO EUROPEIA. European Strategic Plan - i2010 Communication. 2005. Disponível em: <http://bit.ly/2jYAmxS>. Acesso em: 16 ago. 2016. 
MILLARD, J. eGovernment services seen from the user perspective, as part of the eUser project examining evidence-based support for the design and delivery of user-centred online public services. eGovernment report. European Commission IST 6th Framework IST Programme. 2006. Disponível em: <http://www.eusereu.org>. Acesso em: 19 ago. 2016.

NORRIS, P. Digital divide: civic engagement, information poverty and the Internet in democratic societies. New York: Cambridge University, 2001.

ONO, H.; ZAVODNY, M. Gender and the Internet. Social Science Quarterly, Hoboken, v. 84, n. 1, p. 111-121, 2003.

RAGNEDDA, M. The third digital divide: a weberian approach to digital inequalities. London: Routledge, 2017.

RAGNEDDA, M.; MUSCHERT, G. W. Theorizing digital divides and digital inequalities. In: SERVAES, J.; OYEDEMI, T. (Ed.). Social inequalities, media and communication: theory and roots. London: Lexington Books, 2016. p. 23-35.

RAGNEDDA, M.; MUTSVAIRO, B. Demystifying digital divide and digital leisure. In: CARNICELLI, S.; MCGILLIVRAY, D.; MCPHERSON, G. (Ed.). Digital leisure cultures: critical perspectives. New York: Routledge, 2016. p. 107-119.

ROBINSON L. et al. Digital inequalities and why they matter. Information, Communication \& Society, London, v. 18, n. 5, p. 569-582, 2015. Disponível em: <http://www.tandfonline.com/doi/pdf/10.1080/1369118X.2015.1012532>. Acesso em: 18 ago. 2016.

ROGERS, E. M. Diffusion of innovations. 5. ed. New York: Free Press, 2003. 
SELWYN, N.; GORARD, S.; FURLONG, J. Whose Internet is it anyway? Exploring adults' (non)use of the Internet in everyday life. European Journal of Communication, Thousand Oaks, v. 20, n. 1, p. 5-26, 2005.

THIERER, A. D. Is the "digital divide" a virtual reality? Consumers' Research Magazine, Washington, DC, v. 83, n. 7, p. 16-20, 2000.

WARSCHAUER, M. Technology and social inclusion: rethinking the digital divide. Cambridge, MA: MIT, 2003.

WESSELS, B. The reproduction and reconfiguration of inequality: differentiation and class, status and power in the dynamics of digital divides. In: RAGNEDDA, M.; MUSCHERT, G. (Ed.). The digital divide: the internet and social inequality in international perspective. London: Routledge, 2013. p. 17-28.

submetido em: 09 jul. 2016 | aprovado em: 29 ago. 2016 